\title{
A Theory of Non-Universal Laws
}

\author{
Alexander Reutlinger \\ (2011) International Studies in the Philosophy of Science 25, 97-117.
}

\begin{abstract}
Laws in the special sciences are usually regarded to be non-universal. A theory of laws in the special sciences faces two challenges: (I) According to Lange's dilemma, laws in the special sciences are either false or trivially true; (II) they have to meet the Requirement of Relevance, which a way to require the non-accidentality of special science laws. I argue that both challenges can be meet, if one distinguishes four dimensions of (non-)universality. The upshot is that I argue for the following explication of special science laws: $\mathrm{L}$ is a special science law iff (1) L is a system law, (2) L is quasi-Newtonian, and (3) L is minimally invariant.
\end{abstract}

\section{Introduction: Why we need a Theory of Non-Universal Laws}

Many philosophers ${ }^{1}$ are convinced that (fundamental) physics states universal laws, while the special sciences (e.g., biology, psychology, sociology, economics, medical science etc.) state non-universal or ceteris paribus laws (henceforth, cp-laws). ${ }^{2}$ Paradigmatically, Barry Loewer has recently described the important differences between fundamental physical laws (he uses Newton's laws of motion as an example) and special science laws as follows:

"The main relevant differences between fundamental dynamical laws and special science laws are these: The candidates for fundamental dynamical laws are (i) global, (ii) temporally symmetric, (iii) exceptionless, and (iv) fundamental (not furt he r implemented) (v) make no reference to causation. In contrast, typical special science

\footnotetext{
${ }^{1}$ Cf., for instance, Earman and Roberts 1999, Earman, Roberts and Smith 2002, Lange 2000, Roberts 2004, Woodward 2003, 2007, Maudlin 2007, Strevens 2009.

2 Two terminological clarifications: (1) I will use "non-universal laws" and "ceteris paribus laws" interchangeably. (2) My focus is on law statements rather than on laws themselves - thus, my aim is not to argue for any particular metaphysical claim (such as a regularity view, or a dispositionalist account).
} 
laws are (i*) local, (iii*) temporally asymmetric, (iii*) multiply re a lized and implemented, (iv*) ceteris paribus, and ( $\left.\mathrm{v}^{*}\right)$ often specify causal re la tions and mechanisms." (Loewer 2008: $\quad 154$, original emphasis)

In this chapter, I will agree with Loewer and others that the dynamical laws in fundamental physics and the laws in the special sciences differ in the way they describe. ${ }^{3}$ Nonetheless, I will address explicitly only some of the features of special science laws, such as being local, having exceptions or being ceteris paribus. (I will leave aside features such as being temporally asymmetric, the possible multiple realization of special science laws, and the question whether and how fundamental physical laws relate differently to causation than special science laws.) Despite these differences between laws in fundamental physics and laws in the special sciences, most philosophers believe that, in physics as well as in the special sciences, laws are important because they are statements used to explain and to predict phenomena, they provide knowledge how to successfully manipulate the systems they describe, and they support counterfactuals etc. Statements that play these roles in the sciences I call lawish (similarly, Mitchell 1997, 2000 characterizes generalizations in the special sciences as "pragmatic laws" in virtue of performing at least one these roles). Note that, in the debate on laws of nature, lawlikeness is commonly associated with universality (Braithwaite 1959, 301). By contrast, I use "lawish" differently: a general statement is lawish if it is of explanatory and predictive use, successfully guides manipulation, and supports counterfactuals. Contrary to the traditional understanding of laws, being lawish does neither require universality nor other characteristic features of fundamental physical laws. It is certainly a matter of convention whether one would still want to use the term "law" for non-universal general statements. One can either use a new term for lawish, nonuniversal explanatory, general statements (e.g., Woodward and Hitchcock 2003 introduce

\footnotetext{
${ }^{3}$ Many of the problems I will discuss in the paper would be even trickier if one disagreed with Loewer (and others) at this point. Some philosophers (e.g., Cartwright 1983, 1989, Mumford 2004) believe that even fundamental physics deals (at least in part) with non-universal laws. If this were the case, the issue of nonuniversal laws might turn out to be even more pressing.
} 
the term "explanatory generalization"). Or, as I maintain in this paper, one can insist that if a statement plays a lawish role then it shares sufficiently many properties with universal laws in order to be called a law. Christopher Hitchcock and James Woodward admit that their account may be read as a reconceptualization of lawhood (cf. Woodward and Hitchcock 2003, 3).

Let me provide two examples of special science laws. Examples from economics are the law of supply and the law of demand, which - in the words of John Roberts, a critic of cp-laws - state (cf. Roberts 2004, 159, also Kincaid 2004, 177):

Law of Supply: If the supply of a commodity increases (decreases) while the demand for it stays the same, then the price decreases (increases).

Law of Demand: If the demand for a commodity increases (decreases) while the supply remains the same, then the price increases (decreases).

Another example of a lawish statement in the special sciences is the area law in island biogeography:

"the equilibrium number $\mathrm{S}$ of a species of a given taxonomic group on an island (as far as creatures are concerned) increases [polynomially $]^{4}$ with the islands area $[\mathrm{A}]$ : $\mathrm{S}=\mathrm{cA}^{\mathrm{z}}$. The (positive-valued) constants $\mathrm{c}$ and $\mathrm{z}$ are specific to the taxonomic group and island group.” (Lange 2000, 235f; see Lange 2002, 416f.)

Although I lack space to discuss them here, other vividly and controversially debated examples of special science laws are: in neuroscience, the Hodgkin-Huxley Model of the action potential (Weber 2008, 997-1001) and the generalizations describing the mechanism

\footnotetext{
${ }^{4}$ Lange mistakenly writes "exponentially".
} 
of Long-Term Potentiation (Craver 2007, 65-72, 168); in psychology, generalizations describing learning and memory (Gadenne 2004, 107f); in economics, generalizations in models of economic growth (Kincaid 2009, 456f); and in biology, Mendel's law of segregation, the Hardy-Weinberg law, and the principles of natural selection (Beatty 1995, Sober 1997, Mitchell 1997, 2000, Rosenberg 2001, Rosenberg and McShea 2008: 36).

Generalizations like these are believed to be lawish, although they are not universal generalizations. As noted above, traditionally, the most important feature of a law to understand its lawlikeness is universality (cf. Lewis 1973a, 73-76, and Armstrong 1983, 88-93). Furthermore, picturing lawlikeness mainly in terms of universality has lead many theories of causation and explanation to rely on universal laws. This assumption turns out to be problematic: the major challenge for any theory of non-universal laws in the special sciences is to account for their apparent lawish function (in the sense introduced above). If we are not able to provide an explication of non-universal laws, then (at least) the philosophy of the special sciences faces a severe problem concerning causation and explanation in its domains. Many theories of causation and explanation in their standard form presuppose universal laws of nature. ${ }^{5}$ Let me briefly illustrate this point by explaining how most theories of causation in their standard form refer essentially to laws:

Regularity theories of causation hold, roughly, that an event A causes an event B iff, firstly, A preceeds B in time, secondly, A is spacio-temporaly connection to B, thirdly, the proposition that B occurs follows logically from the proposition that A occurs and a law of nature (cf. Mackie 1974, Baumgartner 2008, Strevens 2009).

A proponent of the counterfactual theory, according to David Lewis (cf. Lewis 1973b), decides whether there is a causal connection between two actual events A and B by evaluating the appropriate counterfactuals: (a) "had A been the case, then B would have

\footnotetext{
${ }^{5}$ Interventionist theories of causation rely on lawish generalizations because, if a causal relation between cause A and effect B obtains, a manipulation of the A leads to a change of B that can be described by an invariant generalization (i.e. an explanatory, not necessarily universal, lawlike generalization). Strictly speaking, interventionists insist that generalizations need not be universal. But they also regard this as a challenge: One has to provide a theory of non-universal laws. Cf. Woodward (2003), Woodward and Hitchoock (2003).
} 
been the case", and (b) "had not-A been the case, then not-B would have been the case". According to the standard possible worlds semantics for counterfactuals, this evaluation proceeds by comparing the actual world to similar possible worlds. The most important criterion of similarity relies heavily on universal, fundamental laws of nature (at least in case that the relevant counterfactuals are supposed to be true at the actual world).

According to the transfer or conserved quantity theory of causation (cf. Dowe 2000), causation consists in the possession or the transfer of a physical magnitude (typically a conserved quantity). The transfer or conserved quantity theory of causation builds indirectly on laws of nature: The description of, e.g., energy transfer (or possession of energy) is stated in the language of a physical theory, that contains universal natural law statements - most importantly: conservation laws. ${ }^{6}$

Similarly, many theories of explanation essentially refer to law statements: (1) The DN-model and the IS-model claim that explanation consists in deductive viz. inductive logical inference from laws and initial conditions. (2) Theories of causal explanations inherit their reference to law statements from the concept of causation involved (cf. Lewis 1986, Woodward 2003, Woodward and Hitchcock 2003, Strevens 2009). ${ }^{7}$

The problem stemming from many theories of causation and explanation consists in a logical tension between three assumptions:

(1) The special sciences (a) refer to causes in their domains (i.e. some causal statements in these sciences are true) and (b) provide explanations in their domains.

\footnotetext{
${ }^{6}$ Note that Dowe thinks that conserved quantities are only contingently, not essentially, described by the actual conservation laws. In metaphysics of science, such view can be described as categorialism about conserved quantities (cf. Bird 2007, chapter 3.1). However, at least in the actual world, laws (and especially conservation laws) seem to matter for the truth conditions of causal statements.

${ }^{7}$ Leuridan (2010) argues - correctly, on my opinion - that received accounts of mechanisms and mechanistic explanation (such as Machamer, Darden and Craver 2000, Glennan 2002, Bechtel and Abrahamsen 2005, Craver 2007) also rely on lawish generalizations.
} 
(2) It is a plain fact that the special sciences - possibly, in contrast to physics - cannot rely on universal laws. ${ }^{8}$

(3) Most philosophical theories of causation and explanation - in their standard form essentially presuppose universal laws.

This tension can be formulated as the nomothetic dilemma of causality and explanation (cf. Pietroski and Rey 1995, 85, Woodward and Hitchcock 2003, 2):

First horn: If it is a plain fact that the special sciences cannot rely on universal laws (assumption 2) and if most philosophical theories of causation and explanation essentially involve universal laws and we do not reject these theories (assumption $3)$, then there is no causation and, as well, no explanation in the special sciences (negation of assumption 1).

Second horn: If there is causation and, as well, explanation in the special sciences (assumption 1) and if it is a plain fact that the special sciences cannot rely on universal laws (assumption 2), then there is causation and explanation that does not involve universal laws (negation of assumption 3), i.e. we have to reject the above listed theories of causation and explanation in their standard form.

If we do not want to give up the immensely plausible opinion that the special sciences refer to causes and provide explanations (assumption 1) for purely philosophical reasons, then we are in need of a theory of non-universal laws. So, I will opt for the second horn of the nomothetic dilemma.

\footnotetext{
${ }^{8}$ Cf. Earman, Roberts and Smith (2002, 297f), Woodward (2002, 303), Kincaid (2004), Roberts (2004). As noted above, Cartwright (1983), (1989) and Mumford (2004) dispute the claim that paradigmatic laws of physics conform to the received philosophical picture (e.g. being universal). However, they do not deny that that laws in the special sciences are non-universal, have exceptions etc.
} 
In this paper, I argue as follows: in section 2, I will introduce Lange's Dilemma stating that non-universal laws are either false or trivially true. In section 3, I refer to an attempt to avoid Lange's Dilemma proposed by Paul Pietroski and Georges Rey (1995). Pietroski and Rey attempt to save a cp-law L from being trivially true by explaining counter-instances to L. Although this account has been criticized on good grounds, I think the basic idea is fairly correct. Even its critics, John Earman and John Roberts (1999) among others, sketch a way to repair the account by Pietroski and Rey. Earman and Roberts require that a story has to be told about the relevance of the antecedent for the consequent of a law statement (Requirement of Relevance). I try to fulfill this requirement. In the central section 4, I set up a theory of non-universal laws by distinguishing different meanings (or dimensions) of "non-universal". In section 5, I argue that this approach (a) allows to avoid Lange's Dilemma by conceiving special science laws as quasi-Newtonian, and (b) that it meets Roberts and Earman's Requirement of Relevance by spelling out relevance in terms of invariance. In section 6 , I argue that the results of the preceding sections amount to this theory of special science laws: L is a special science law iff (1) L is a system law, (2) L is quasi-Newtonian, and (3) L is minimally invariant.

\section{Challenge I: Falsity and Triviality}

A philosophical reconstruction of lawish statements in the special sciences faces a severe problem, which can be articulated in the form of Lange's Dilemma (cf. Lange 1993, 235). Here is the first horn:

First horn (Falsity): Strictly and literally speaking, special science laws are false because it is not the case that all Fs are Gs (if that is what the laws say).

For instance, the relationship between supply and price is not always as the law of supply says (or, as it seems to say prima facie), because an interfering factor might occur. In other 
words, special science laws that instantiate perfect regularities are - mildly put "scarce" (Cartwright 1983, 45). Yet, if one supposes that the law is to be formalized as a universally quantified conditional sentence. Then one counter-instance (due to a disturbing factor) to the universally quantified sentence means that it is false.

The second horn of Lange's Dilemma can be stated as follows:

Second horn (Triviality): If laws in the special sciences are cp-laws, then they are trivially true.

If we suppose that an implicit cp-clause is attached to the law then it seems to be equivalent with "All Fs are Gs, if nothing interferes". But then the cp-law in question is in danger to lack empirical content. It lacks empirical content because it seems to say nothing more than "All Fs are Gs or (it is not the case that all Fs are Gs)". Note that the second horn of Lange's dilemma seems to depend on the exclusive reading of the ceteris paribus clause ("if nothing interferes", "if all disturbing factors are absent"). If this were the correct theory of laws in the special sciences, then these laws would be analytically true sentences and, therefore, trivially true. Obviously, this is a bad result because laws of the special sciences should be reconstructed as (approximately) true empirical statements - not as sentences being true in virtue of the meaning of their components. Note that the second horn is a more pressing problem than the first, because I have already given up the assumption that special science laws are universal (as presupposed in the first horn) - to be precise, I reject the claim that special science laws are universal in two readings of "universal" (see Section 4). In the recent debate, some philosophers take Lange's Dilemma as a reason to be pessimistic about whether there really is a convincing explication of laws in the special sciences:

,$[\ldots]$ there is no persuasive analysis of the truth conditions of such laws; nor is 
there any persuasive account of how they are saved from vacuity; and, most distressing of all, there is no persuasive account of how they meld with standard scientific methodology, how, for example, they can be confirmed or disconfirmed. In sum, a royal mess.“ (Earman and Roberts 1999: 470f, my emphasis)

So, to deal with this dilemma is clearly a central semantic challenge, which also has epistemological consequences as Earman and Roberts point out: in order to be empirically testable, special science laws have to be true (contrary to the first horn) and non-trivial (contrary to the second horn). In this paper, I will restrict my attention to the semantic challenge posed by Lange's dilemma.

\section{Challenge II: Requirement of Relevance}

Pietroski and Rey $(1995,92)$ claim that it is sufficient for a law "cp, $\forall(x) A x \rightarrow C x$ " to avoid Lange's Dilemma (i.e. to be neither necessarily false nor trivially true) if the following conditions are satisfied:

(1) A and $\mathrm{C}$ are nomological predicates. ${ }^{9}$

(2) Assessing a law statement $\mathrm{L}$ "cp, $\forall(\mathrm{x}) \mathrm{Ax} \rightarrow \mathrm{Cx}$ " leads to a commitment which is expressed by the Explanatory-Commitment-Condition (ECC): If a counter-instance $(\mathrm{A} \wedge \neg \mathrm{C})$ to law statement $\mathrm{L}$ occurs, then one is committed to explain $\neg \mathrm{C}$ by referring to a factor $\mathrm{H}$ which is independent of L. Pietroski and Rey allow two possibilities w.r.t. the independent explanatory force of $\mathrm{H}$ : (i) $\mathrm{H}$ alone explains $\neg \mathrm{C}$, or (ii) $\mathrm{H}$ in conjunction with $\mathrm{L}$ explains $\neg \mathrm{C}$.

(3) It is the case that either (i) $A \wedge C$, or (ii) $A \wedge \neg C$ and ECC is satisfied.

\footnotetext{
${ }^{9}$ Pietroski and Rey $(1995,92)$ argue that A and C are not "grue-like".
} 
According to Pietroski and Rey $(1995,90), \neg \mathrm{C}$ is explained independently of L by referring to $\mathrm{H}$ if (a) $\mathrm{H}$ is not a logical consequence of L (i.e. logical independence of $\mathrm{H}$ ), and (b) the explanatory factor $\mathrm{H}$ is not an effect of $\neg \mathrm{C}$ (i.e. causal independence of $\mathrm{H}$ ). The critics of this approach have argued that ECC is not sufficient for saving special science laws from Lange's Dilemma, because ECC allows that (1) A is completely irrelevant for C and (2) $\neg \mathrm{C}$ is still perfectly explained independently of L by a factor H (cf. Earman and Roberts 1999, 453f; Schurz 2001, 366f; Woodward 2002, 310). Earman and Roberts provide the following counterexample:

"Unfortunately, [Pietroski and Rey's proposal] is not sufficient for the nonvacuous truth of the cp-law. To see why, let "Fx" stand for "x is spherical", and 1 e $t$ "Gy" stand for $\mathrm{y}=\mathrm{x}$ and $\mathrm{y}$ is electrically conductive". Now, it is highly plausible that for any body that is not electrically conductive, there is some fact about it namely its molecular structure - that explains its non-conductivity, and that this fact also explains other facts that are logically and causally independent of its nonconductivity - e.g., some of its thermodynamic properties. Thus, clauses (ii) and (iii) [i.e. the conditions (2) and (3) of Pietroski and Rey's account above] appear to be easily satisfied. If Pietroski and Rey's proposal were correct, then it would follow that ceteris paribus, all spherical bodies conduct electricity." (Earman and Roberts 1999, 453, emphasis added)

Earman and Roberts comment on their counterexample:

"The general moral of this observation seems to be that it is not enough simply to require, $[\ldots]$ that when $\mathrm{cp}:(\mathrm{A} \rightarrow \mathrm{B})$, any case of $\mathrm{A}$ accompanied by $\sim \mathrm{B}$ must be such that there is an independent explanation of $\sim \mathrm{B}$. This is because this requirement does not guarantee that $\mathrm{A}$ is in any way relevant to $\mathrm{B}$, which surely must be the case if cp: 
$(\mathrm{A} \rightarrow \mathrm{B})$ is a law of nature. Perhaps Pietroski and Rey's proposal could be modified to remedy this defect. (Earman and Roberts 1999, 454, my emphasis)

My theory of non-universal laws is an attempt to explain what "relevance" means and to save (a version of) ECC at the same time. I agree with the critics that ECC cannot be sufficient. But I will argue that Pietroski and Rey basically had the right idea. More precisely, I will argue in a twofold manner: On the one hand, it is correct that something close to ECC is necessary for a theory of special science laws. It is necessary for dealing with disturbing factors. I will rely on quasi-Newtonian laws for these purposes (see section 4.2). On the other hand, Earman, Roberts, Schurz, and Woodward are completely justified to demand that we have to account for the relevance of the antecedent for the consequent of a law statement. I will provide such an account of relevance in terms of invariance (see section 4.3).

\section{Four Dimensions of Non-Universal Laws}

As I argued in the introduction, it is the received view that, in the special sciences, laws appear to be non-universal - or, they are said to 'have exceptions'. But what does it mean to be universal, and, respectively, non-universal? Surprisingly, in the recent debate on cplaws this question is not answered in a systematic way. ${ }^{10}$ The lack of a systematic approach is a major problem, because universality is an ambiguous concept. In accord with Andreas Hüttemann (2007, 139-141), we may distinguish four meanings or dimensions of universality with respect to a law statement:

(1) First Dimension - Universality of space and time: Laws are universal ${ }_{1}$ iff they hold for all space-time regions.

\footnotetext{
${ }^{10}$ Notable exceptions are Mitchell (2000) and Schurz (2002).
} 
(2) Second Dimension - Universality of Domain of Application: Laws are universal 2 iff they hold for all (kinds of) objects.

(3) Third Dimension - Universality for External Circumstances: Laws are universal 3 iff they hold under all external circumstances (i.e. circumstances that are not referred to by the law statement itself). ${ }^{11}$

(4) Fourth Dimension - Universality with respect to the Values of Variables: Laws are universal $_{4}$ iff they hold for all possible values of the variables ${ }^{12}$ in the law statement. Universality in this sense acknowledges that laws usually are quantitative statements (and, thus, the predicates contained in these statements are to be conceived as variables ranging over a set of possible values).

Paradigm examples of fundamental physical laws (such as Newton's laws, Einstein's field equations, and the Schrödinger equation) are usually taken to be universal in all four dimensions (cf. Schurz 2002, section 6.1; Hüttemann 2007, 139-141). I will argue that lawish generalizations in the special sciences are (a) universal in the first and second dimension and (b) non-universal with respect to the third and the fourth dimension of

11 A useful way to spell out the third dimension of universality could be found in Loewer's use of "global" (see the quote in the introduction): "The dynamical laws of classical mechanics are complete and deterministic. Given the state at any time $t$ they determine the state at any other time. The determination is global since the position and momentum of any particle at a time $\mathrm{t}+\mathrm{r}$ is determined only by the global (i.e. the entire) state of that system at time $t$. That is, to know how any one particle moves at $t+x$ one has to know something at each particle at $\mathrm{t}$. The dynamical laws and a partial description of state at $\mathrm{t}$ (except in special cases) do not entail much about the state of the system at other times and, in particular, don't say much about what any particular particle will (was) doing at $\mathrm{t}+\mathrm{r}$." (Loewer 2008, 155) In contrast with the laws of classical mechanics, a special science law (such as the law of supply) is non-global, incomplete, and, thus, seems to provide only a "partial description" of the phenomenon it describes. Special science laws leave out other influences on the phenomenon i.e. circumstances that are not referred to by the law statement itself - as stated in the description of the third dimension of universality (cf. Pietroski and Rey 1995, 89).

${ }^{12}$ A variable $X$ (in the terminology of statistics and causal modeling) is a function $X: D \rightarrow \operatorname{ran}(X)$, with a domain $\mathrm{D}$ of possible outcomes, and the range $\operatorname{ran}(\mathrm{X})$ of possible values of $\mathrm{X}$. For quantitative variables $\mathrm{X}$, $\operatorname{ran}(\mathrm{X})$ is usually taken to be the set of real numbers (cf. Pearl 2000; Eagle 2010, Chapter 0.9). For example, temperature is represented by a variable $\mathrm{T}$ that has several possible values such as $\mathrm{T}=30,65^{\circ}$. However, in the debate on causation philosophers often use qualitative, binary variables with $\operatorname{ran}(X)=\{0 ; 1\}-$ whether a binary variable takes one of its values is taken to represent whether or not a certain type of event occurs (cf. Hitchcock 2001). On notation: capital letters, such as X, Y, .., denote variables; lower case letters, such as X, $\mathrm{y}, \ldots$, denote values of variables; the proposition that $\mathrm{X}$ has a certain value $\mathrm{x}$ is expressed by a statement of the form $X=x$, i.e. $X=x$ is a statement about a event-type (cf. Woodward 2003). 
universality. This diagnosis amounts to a challenge: any theory of lawish generalizations in the special sciences is obliged to explain how a lawish statement can be non-universal $\& 4$ and still play a lawish role.

\subsection{Universality 1 \& : System Laws}

Are special science laws universal in the first and the second dimension of universality? I think, the answer is yes. I will argue for two claims: first, lawish generalizations in the special sciences hold for all space-time regions (i.e. they are universal 1 ), however these generalizations simply lack application in some space-time regions. Secondly, lawish statements in the special sciences can be reconstructed in a way that they do not quantify over a restricted domain of objects (i.e. they are universal 2 ). Arguing for these claims might not seem plausible at first glance, because usually generalizations in the special sciences are interpreted as system laws. Gerhard Schurz (2002, Section 6.1) has introduced the notion of a system laws: while fundamental physical laws "are not restricted to any special kinds of systems (be it by an explicit antecedent condition or an implicit application constraint)“" (Schurz 2002, 367), system laws refer to particular systems of a certain (biological, psychological, social etc.) kind $K$ in a specific space-time region. Hence, so the usual characterization continues, lawish statements in the special sciences typically have an in-built historical dimension which the fundamental physical laws lack, because they are restricted to a limited space-time region where the objects of a certain kind $K$ exist (for instance, cf. Beatty 1995, Rosenberg 2001). ${ }^{13}$ I will argue that Schurz is absolutely correct in characterizing lawish statements in the special sciences as being "restricted to [...] special kinds of systems (be it by an explicit antecedent condition or an implicit application constraint)“ (Schurz 2002, 367) - however, if one adopts this

\footnotetext{
${ }^{13}$ Note that the concept of a system in Schurz's sense seems to at least conincide with the use of the concept system in the literature on mechanistic explanation in the life sciences (cf. Machamer, Darden and Craver 2000, Glennan 2002, Bechtel and Abrahamsen 2005, Craver 2007). Mechanists usually conceive a system to be composed of interacting parts. Schurz (2002, Section 5) seems to agree with this characterization of a system when he discusses examples of biological systems.
} 
characterization of special science laws as system laws, then one is still not committed to deny that these law statements are universal in the first and second dimension.

Does this characterization of system laws mean that special science laws are nonuniversal ${ }_{1}$ ? No. Simply because a generalization $G$ does not have an application in some space-time region $s$, it does not mean that the law does not hold at $s$. In order to be truly non-universal 1 , G would have to be conform to a thought experiment of "Smith's Garden" by Tooley:

"All the fruit in Smith's garden at any time are apples. When one attempts to take an orange into the garden, it turns into an elephant. Bananas so treated b e c o m e apples as they cross the boundary, while pears are resisted by a force that cannot be overcome. Cherry trees planted in the garden bear apples, or they bear nothing at all. If all these things were true, there would be a very strong case for its being a law that all the fruit in Smith's garden are apples. And this case would be in no way undermined if it were found that no other gardens, however similar to Smith's garden in all other respects, exhibited behaviour of the sort just described." (Tooley 1977, 686, my emphasis)

So, according to Tooley, a law L can be spacio-temporally restricted to a space-time region $s$ (as the laws in Smith's garden) in the sense that L fails to be true in a situation that is perfectly similar to the situation in $s$, except for the fact that this perfectly similar situation is located in a different space-time region $s^{*}$.

I think that laws that are truly non-universal ${ }_{1}$ would be similar to the laws that true of various fruit in Smith's garden. But it seems to be a far too strong claim that laws in the special sciences are local in a way as the laws in Smith's garden are. Thus, it seems to be a more promising option to say that (a) special science laws are universal 1 and (b) these laws simply lack application in some space-time regions. For instance, to say that the law of 
supply does not hold on mars because there are no people buying and selling goods does not indicate that the law of supply is a local law. A better understanding seems to be that the law of supply factually has no application on Mars (or, it lacks instances on Mars).

Does this characterization of lawish statements in the special sciences as system laws mean that special science laws are non-universal 2 ? No. At first glance, special science laws, if viewed as system laws, appear to be non-universal 2 : special science laws quantify over a restricted domain of objects of a certain kind - not over a domain of objects of all kinds. For instance, the law of supply seems to quantify over the restricted domain of commodities - not over an unrestricted domain. So, one might get the idea that the domain of a special science is a restricted domain. The law of supply can be formalized as quantifying over a restricted domain $C$ of commodities (with $c$ as an individual variable of this domain):

$$
\text { “ } \forall(c)((\text { supply increases }) c \wedge(\text { demand constant }) c) \rightarrow(\text { price decreases }) c " .14
$$

But is this really a convincing reconstruction of lawish statements in the special sciences? There is an alternative formalization that quantifies over a domain of all objects. This formalization interprets the kind of object (here: commodities) as a predicate and not as a restriction of the domain (with $x$ as an individual variable for the unrestricted domain):

$$
" \forall(x)((\text { commodity }) x \wedge \text { (supply increases }) x \wedge(\text { demand constant }) x) \rightarrow \text { (price }
$$
decreases) $x "$.

\footnotetext{
${ }^{14}$ One could object that even if the restricted reading were the favored reading it would not be clear why the corresponding universal statement should be true. Even the universal statement (quantifying only over commodities) is vulnerable to Lange's dilemma and the Requirement of Relevance. The lesson, I think, we should learn from this result is that the responses to these two challenges have to given w.r.t. to the third and fourth dimension of non-universality.
} 
The second, unrestricted formalization of the law of supply is a way to save universality2. Formalizing special science laws in this form, reconstructs them as laws that hold for all objects..$^{15}$

Obviously, I do not offer a theory for the first and second dimension of universality. Such a theory would have to elucidate why generalizations that are universal ${ }_{1 \& 2}$ are lawish. All I did was to provide a reconstruction such that special science laws can be consistently understood as being universal $1_{122}$. This is not a trivial result, because philosophers, such as Beatty (1995) and Rosenberg (2001), insist that generalizations in the biological and the social sciences should be regarded as (a) being historical in the sense of applying only to a specific space-time region (this is in contradiction with universality 1 ), and (b) as referring to a restricted domain of objects (this contradicts universality 2 ). Contrary to these philosophers, I merely wanted to point out that one can maintain that lawish generalizations in the special sciences are universal w.r.t. the first and the second dimension of universality. It is a matter of convention to still call these lawish statements "system laws" in order to highlight difference to fundamental laws.

In the following sections, I will argue that general statements play a lawish role, if they can deal with disturbing factors (dimension 3), and if they are invariant under different possible initial conditions (dimension 4). In other words, all the work is to be done by theories of non-universality $3 \& 4$.

\subsection{Non-Universality 3 : The Method of quasi-Newtonian Laws}

How shall we deal with the third dimension, i.e. the fact that special science laws are sensitive to external factors? Recall that the second horn of Lange's dilemma presupposes a reconstruction of law statements that are qualified by only a cp-clause like "all disturbing

\footnotetext{
${ }^{15}$ An anonymous referee has pointed out that one might want to dispute the claim that even the fundamental laws do not apply to everything (contra Schurz 2002, Hüttemann 2007). S/he argues that the fundamental laws, for instance, do not apply to angels and numbers. However, I think that, even if this were the case, we could preserve the universality2 for the fundamental laws by exactly the same strategy which I just used for preserving universality 2 for lawish statements in the special sciences. Further, my arguments do not have to rely on the characterization of fundamental physical laws which Schurz and Hüttemann provide.
} 
factors are absent". But one is not committed to this reading. Is there an alternative reconstruction? My positive thesis is:

Positive Thesis: A special science law $\mathrm{L}$ is backed up by a distinct law $\mathrm{L}^{*}$ which describes (comparatively or quantitatively) the influence of relevant disturbing factors.

In order to argue for my positive thesis, I will rely on the concept of a quasi-Newtonian law (I follow the name that Tim Maudlin 2004 has used, see below). The basic idea is that factors that lead to a counter-instance of the law L are described by other laws L*. My positive thesis is intuitively supported the fact that laws are typically not isolated but part of a theory or a model. Some disturbing factor with respect to law $L$ is often described by law $L^{*}$ in the same theory or model. The positive thesis is a version of ECC. The claim that the influence of a disturbing factor (for a lawish statement L) is described by another law $L^{*}$ is fairly close to (or a special case of) explaining the counter-instances of $L$ by an independent factor - as ECC states. Yet, ECC and quasi-Newtonian laws differ in at least in one important respect: while Pietroski and Rey's ECC seems to aim at epistemic acceptability conditions of a cp-law, quasi-Newtonian laws provide non-epistemic and perfectly objective truth-conditions for laws in the special sciences.

Historically, this key idea of dealing with disturbing factors has been proposed by John Stuart Mill:

"The disturbing causes have their laws, as the causes which are thereby disturbed have theirs; and from the laws of disturbing causes, the nature and amount of the disturbance may be predicted a priori, like the operation of the more general laws which they are said to modify or disturb, but with which they might more properly said to be concurrent." (Mill 1836/2008, 50, my emphasis) 
For instance, the law of supply states "If the supply of a commodity increases (decreases), then the price decreases (increases)". It is usually added to the antecedent "... while the demand for this commodity stays the same" which implies that the law of supply does not hold if the demand increases or decreases. At this point it is crucial to notice that the evolution of the price of a good is not described by a single generalization, i.e. by the law of supply. The evolution of the price also depends on another factor, the demand of a good, described by the law of demand: "if the demand for a commodity increases (decreases) while the supply remains the same, then the price increases (decreases)". It has to be emphasized that the equilibrium model of supply and demand also describes what would happen, if the demand does not remain the same. In other words, the evolution of the price of a commodity is described by an equilibrium model, according to which supply and demand can vary independently (Hausman 1992; Mas-Colell, Whinston and Green, 1995).

In order to illustrate Mill's original idea how a disturbing factor can also be described by a law, I draw on Maudlin's (2004) concept of a quasi-Newtonian law. In Newton's physics, Newton's First Law describes the inertial behavior of a physical system: the uniform motion of a system when no force acts upon it. Newton's Second Law describes the deviant behavior: the change of inertial motion if other forces are present. Maudlin characterizes the general form of Quasi-Newtonian Laws by analogy to Newton's laws:

"Let us denominate laws quasi-Newtonian if they have this form: There are, on the one hand, inertial laws which describe how some entities behave when nothing acts on them, and there are laws of deviation that specify in what conditions, and in what ways, the behavior will deviate from the inertial behavior." (Maudlin 2004, 431, my emphasis) 
Maudlin $(2004,434)$ stresses that special science laws are typically quasi-Newtonian. So, let us apply Maudlin's idea to the economic case: the law of demand describes inertial behavior; if the law of supply is integrated in the equilibrium model, then the whole model describes the deviant behavior (of the price). Thus, the laws of demand and supply describing the evolution of the price of a commodity are a quasi-Newtonian law. As Lange observes, the case is analogous concerning the area law in island biogeography: it matters how far an island is away form the coast - being very far away might count as a disturbance of the area law. Island bio-geographers describe this disturbing factor with the distance law: "ceteris paribus, islands farther away from the mainland equilibrate at lower biodiversity levels" (Lange 2002, 419). In Maudlin's terminology, we might call a model including the distance law a law of deviation (with respect to the area law as an inertial law) a quasi-Newtonian law of island bio-geography. In a recent paper, Craig Callender and Jonathan Cohen draw a similar analogy between Newton's laws of motion and the way in which the special sciences (they focus on Malthus's exponential law in ecology) describe the deviation w.r.t. one laws by other laws:

"Consider the so-called first principle of population dynamics, Malthus's exponential law: $\mathrm{P}(t)=\mathrm{P}_{0} \mathrm{e}^{r t}$, where $\mathrm{P}_{0}$ is the initial population (say, of rabbits), $r \mathrm{t} \quad \mathrm{h} \quad \mathrm{e}$ growth rate, and $t$ the time. This ecological generalization is very powerful. It supports counterfactuals and crucially enters ecological predictions and explanations. It has an undeniably central role in most presentations of the science of ecology. Indeed, it arguably has the very same central role in ecology that Newton's first law does in classical mechanics: both express a kind of ideal default behavior - exceptions to which are to be explained with further laws (Ginzburg and Colyvan, 2004). In short, pending some good reason for distinguishing Malthus's Law over Newton's first law, there is good reason for taking seriously the idea that the former should count as a bona fide law of nature. (If you don't like 'law of nature', substitute 'widely applicable 
projectible generalization' in its stead.)"

(Callender and Cohen 2010, 427, my emphasis)

Maudlin calls these laws quasi-Newtonian for a good reason, because there are important disanalogies to Newton's laws and special science laws (if understood in terms of quasiNewtonian laws):

First, in the economic case (as well as in the island bio-geographical and the ecological case) it depends on pragmatic choice whether the law of supply or the law of demand is dubbed "inertial law". ${ }^{16}$ The important point for our purposes is that a larger model in which the inertial law is integrated (e.g. the equilibrium model of a market) describes that deviating behavior of the price evolution - that is, the behavior is deviating relative to a chosen inertial law such as the law of supply.

Second, in cases of special science laws the deviation laws are usually not universal $_{1-4}$ as Newton's Second Law is.

Third and most importantly, those disturbing factors governed by the laws of deviation fall into two classes: those that are in the scope of a particular discipline and those that are not. For a special science, like economics, there will always be disturbing causes (such as comets) which will not be integrated in the models of this discipline. Concerning these latter factors, we are committed to an existential claim if we want to maintain that special science laws are quasi-Newtonian: if a special science system deviates from its inertial behavior, then there are (known or unknown) laws of deviation describing the influence of disturbing factors on the inertial behavior. ${ }^{17}$ The examples introduced in the preceding paragraph provide a good reason to believe that this existential

\footnotetext{
${ }^{16}$ A typical example is provided by causal models in econometrics: according to these models, the causal influence of a variable in isolation is described by a single structural equation. Each one of those single equations might be called "inertial law". However, the whole causal model (i.e. a set of equations) provides an overall output resulting from the interaction of various causal factors (cf. Cartwright 1989, Chapter 4.5; Pearl 2005, Chapter 5).

${ }^{17}$ Thanks to Tim Maudlin and Michael Strevens for suggesting this amendment.
} 
claim is likely to be true, because the influence of disturbing factors within a specific special science (e.g. micro-economics, bio-geography, ecology) is indeed described by a law of deviation. Since the scope of a special science is limited (e.g. influence of comets crashing on Earth is not described within economics) an advocate of the quasi-Newtonian approach has to make the amendment that there are - unlike in the case of Newtonian laws of motion - unknown disturbing factors and unknown corresponding laws of deviation from the point of view of a special science like economics. Michael Strevens (2010, section 3) argues for a similar point: the conditions of application of a special science law are partly "opaque" for the researchers, say economists, because the researchers lack complete knowledge of all disturbing factors and the laws of deviation governing them. However, I agree with Strevens that the fact that economists have incomplete knowledge does not imply that the existential claim about unknown disturbing factors and unknown corresponding laws of deviation is false.

Despite these disanalogies, I think that the positive analogy remains intact: Some laws in physics and in the special sciences are quasi-Newtonian because the influence of a disturbing factor on a system describes by a law L is described (comparatively or quantitatively) by another law $\mathrm{L}^{*}$.

Relying on quasi-Newtonian laws raises a question: Do scientists need to know all the laws of deviation describing the influence of all possible disturbing factors? Are all (possible) disturbing factors equally important? And, if this is not the case: how does one distinguish important disturbing factors from irrelevant ones? Intuitively speaking, it seems quite obvious (and descriptively adequate) that scientists are not interested in all possible disturbing factors. Rather, scientists seem to discriminate relevant and (more or less) irrelevant disturbing factors. Marc Lange (2000) provides a convincing pragmatic answer to the questions asked above. According to Lange's core idea, laws in the special sciences are propositions whose application is pragmatically restricted to the purposes of a scientific discipline (Lange 2002, 416). Lange identifies several strategies which scientists 
use in order to distinguish relevant disturbing factors from irrelevant ones. For instance, Lange discussed the strategy of non-negligibility: instead of providing a complete list of all interfering factors, scientists merely refer to those interfering factors "that arise sufficiently often, and can cause sufficiently great deviations from $G$-hood, that a policy of inferring $F \mathrm{~s}$ to be $G[\ldots]$ would not be good enough for the relevant purposes" (Lange 2002, 411; Lange 2000, 170f). For instance, consider the economic law of supply. According to Lange, it may happen that the increase in supply is so small that no decrease in prize results. It depends on the goals of the researchers in questions whether this description of the increase in supply is fine-grained enough. It might also happen that the price does not decrease although the supply increases significantly, because a gigantic comet hitting the planet Earth and destroying all life on its surface disturbs the instantiation of this law. The comet causes sufficiently great deviation from a decrease in the price of a good. Nevertheless comets are negligible for the purposes of economists because their occurrence does not arise sufficiently often to count as interfering factor that is to be explicitly listed in the cp-conditions of the law of supply.

The lesson we can learn from Lange is that, ontologically speaking, all of the disturbing factors (and the corresponding laws of deviation) are on a par. However, from a pragmatic point of view, it seems to be the case that scientists rank the relevance of disturbing factors with respect to the aims of the research in their particular fields. If one follows Lange, then one way to describe this ranking is, for instance, the strategy of nonnegligibility. If one considers this strategy to be sensible reconstruction of a methodology implicit in scientific practice, then only those disturbing factors that are evaluated as relevant in the light of this strategy have to be described by laws of deviation.

\subsection{Non-Universality4: Invariance}


My earlier diagnosis was that special science laws might fail to hold for all possible values of the variables in the law statement. That is, they are non-universal4. According to invariance theories of laws, a generalization may be non-universal 4 but nonetheless lawish. The general idea of invariance theories of laws consists in the claim that only laws remain true for different possible initial conditions which are taken to be results of interventions (I return to this issue in more detail below). A generalization is invariant if it holds for some, possibly limited, range of the possible values of variables (expressing initial conditions which are taken to be results of interventions). For instance, the law of demand might be true for various possible units of a commodity that are demanded, but it might not hold for extremely high amounts of demand (e.g., for a demand of 30 Billion units of any commodity, this feature is often referred to as the elasticity of the price). The decisive question is: Can special science laws be lawish and still be non-universal 4 ? I think that invariance theories of laws provide a positive answer to this question. Furthermore, I argue that invariance theories have an additional benefit: they satisfy the Requirement of Relevance, because relevance can be spelled out as invariance (I will turn to this issue in section 5). I will start by using Woodward and Hitchcock's invariance theory of laws. ${ }^{18}$ Then I will propose a revised version of an invariance theory, because Woodward and Hitchcock's theory crucially depends on the notion of possible intervention which is regarded to be problematic by some philosophers (see below).

According to Woodward and Hitchcock $(2003,17)$ and Woodward $(2003,250)$ a statement $G$ is minimally invariant iff the testing intervention condition holds for $G$. The testing intervention condition states for a generalization $\mathrm{G}$ of the form $\mathrm{Y}=f(\mathrm{X})$ :

(1) there are at least two different possible values of an endogenous variable $X, x_{1}$ and $\mathrm{x}_{2}$, for which $\mathrm{Y}$ realizes a value in the way that the function $f$ in $\mathrm{G}$ describes and

\footnotetext{
18 Similar approaches such as Lange's $(2000,103 ; 2009,29)$ and Mitchell's (2000) stability theories as well as Ladyman and Ross's (2007) “real pattern”-approach might also work for my argument.
} 
(2) the fact that $\mathrm{X}$ takes $\mathrm{x}_{1}$ or, alternatively, $\mathrm{x}_{2}$ is the result of an intervention.

Let me first turn to part (1) of the testing intervention condition. The most intuitive case of a testing intervention might be the following one: $\mathrm{X}=\mathrm{x}_{1}$ describes an actual state of affairs while $\mathrm{X}=\mathrm{x}_{2}$ describes a possible counterfactual state of affairs. For instance, suppose that the ideal gas law $-\mathrm{pV}=\mathrm{NkT}-$ is true for the actual temperature of a gas $g$ of $30^{\circ}$. According to the first part of the testing intervention condition, the ideal gas law is minimally invariant if it also holds for a (counterfactual) temperature of, say, $40^{\circ}$. Analogously, the area law and the law of supply conform to the first part if these laws remain true for at least two values of the independent variables (i.e. the island area variable and the supply variable).

Part (2) of the testing intervention condition specifies and restricts the type of situation in which part (1) is supposed to hold: it is a (counterfactual) situation in which the behavior described by the generalization in question is undisturbed by external factors. For instance, in the case of the law of supply one considers a situation in which the demand stays constant (that is, the demanded quantity of a commodity is in the "redundancy range", i.e. the demand is held constant such that the price is not influenced by the demand, if the supply does not change, cf. Woodward 2003, 83; Hitchcock 2001, 290). Given such a situation, one changes the values of a variable as indicated by part (1) of the testing intervention condition. Woodward and Hitchcock prefer to describe the change of a variable causally: an intervention causes the change of a variable in a (counterfactual) situation in which the behavior described by the generalization in question is undisturbed by external factors in the sense that disturbing causes are held constant in the redundancy range (for a precise definition of intervention variables and interventions cf. Woodward 2003: 98).

Yet, I think that the reference to interventions is by no means necessary in order to state what invariance is (see Mitchell 2002: $346 \mathrm{f}$ for a similar view). Let me sketch an 
alternative: analogously to the testing intervention condition, consider a (counterfactual) situation in which the behavior described by the generalization in question is undisturbed by external factors in the sense that these factors take values in the redundancy range. Call such a situation an "isolation situation". Equipped with this terminology, one can refine minimal invariance without reference to interventions (as Woodward and Hitchcock understand the concept):

A statement $G$ of the form $\mathrm{Y}=f(\mathrm{X})$ is minimally invariant iff there are two values of $\mathrm{Y}$ that counterfactually depend on distinct values of $\mathrm{X}$ in an isolation situation, i.e. a situation in which other causes take values in the redundancy range.

It might be a great advantage to be able to define minimal invariance purely in terms of counterfactual dependence in situations in which other factors are held fixed ${ }^{19}$, because there are good reasons to believe that the notion of an intervention is itself problematic. ${ }^{20}$

What is the pay-off of invariance theory? Invariance theory does not require lawish statements to be universal4. Thus, adopting invariance theory seems to be $a$ plausible way to account for the lawishness of generalizations that are non-universality 4 . Furthermore,

\footnotetext{
${ }_{19}$ My approach does not differ from Hitchcock and Woodward's invariance theory concerning the nonreductive feature of the explication of the concept of a lawish generalization. Both explications are nonreductive, because they use causal and nomological concepts in the explicans. I agree with Hitchcock and Woodward that the non-reductive character of an explication is unproblematic as long as the explication is not viciously circular. For a more detailed defence of non-reductive explication see Woodward (2003: 103f, 2008: 203f), Strevens (2008: 186), Reutlinger (forthcoming).

${ }^{20}$ For instance, Strevens (2007), (2008) argues that interventions lead to an infinite regress problem; and Reutlinger (in print) argues that the modal character of possible interventions (i.e. logical possibility, as assumed by Woodward 2003: 131f) leads to severe trouble when merely logically possible (and physically impossible) interventions figure in counterfactuals - as interventionists claim.
} 
invariance theories are a promising tool to meet the Requirement of Relevance (see Section $5) .^{21}$

\section{Meeting the Challenges: Lange's Dilemma and the Requirement of Relevance}

How does the distinction of four dimensions of non-universality help to meet the challenges? Accepting the universality in the first and second dimension (i.e., we may say that special science laws are system laws), the theories of the third and the fourth dimension solve the problems. How? Lange's Dilemma addresses a problem of generalizations that are context-sensitive with respect to disturbing factors. Naturally, I claim that our theory of the third dimension has to deal with Lange's Dilemma: quasiNewtonian laws are describing the influence of disturbing factors. The Requirement of Relevance addresses a problem concerning the relation between the antecedent and consequent of the law statement. In terms of variables, the Requirement of Relevance addresses a problem concerning the question whether a law statement holds for different possible value of the antecedent variable(s). Naturally, I claim that our theory of the fourth dimension has to deal with the Requirement of Relevance: relevance is understood as invariance. I will now argue that, first, Lange's Dilemma can be avoided, and, second, that the Requirement of Relevance can be fulfilled.

First, does adopting the view that lawish statements in the special sciences are quasi-Newtonian laws avoid Lange's Dilemma? I argue that it does: quasi-Newtonian laws describe the influence of disturbances. Assuming that laws are quasi-Newtonian avoids Falsity because the occurrence of a disturbance does not render the law $\mathrm{L}$ in question false - instead the influence of a disturbing factor is described by another law $\mathrm{L}^{*}$ (and $\mathrm{L}^{*}$ might be known or unbeknown by scientists). It avoids Triviality because it is not (exclusively)

\footnotetext{
${ }^{21}$ As a referee pointed out, invariance theories usually refer to counterfactual situation in which the factors governed are undisturbed (i.e. the counterfactual situation involves elements of idealization and abstraction) in order to state the truth-conditions of law statements. A methodological question immediately comes to mind: how can one test these statements? How do scientists actually test statements of this kind in practice? Various authors have addressed these questions (cf. Kincaid 1996, Cartwright 2002, Steel 2007, Reiss 2008, Hüttemann forthcoming).
} 
committed to the fatal expression "if nothing interferes" - rather quasi-Newtonian laws describe two kinds of situation: undisturbed (i.e. "inertial") behavior and disturbed ("deviant") behavior of a kind of system. I argued that an advocate of the quasi-Newtonian approach is committed to the following existential claim: there are known and unknown disturbing factors, and know and unknown corresponding laws of deviation from the point of view of a special science.

Second, according to Earman and Roberts (1999), explaining why a counterinstance to L occurred is not enough to avoid Lange's Dilemma - what has to be supplemented is an account of relevance. I called this the Requirement of Relevance. I think that being invariant nicely meets the challenge, because relevance is spelled out in terms of counterfactual dependence between the possible values of those variables figuring in $\mathrm{L}$. In other words, the antecedent $\mathrm{A}$ of a law $\mathrm{L}$ is relevant for the consequent $\mathrm{C}$, if $\mathrm{L}$ is invariant (i.e., L holds for at least one alteration of $\mathrm{A}$ in a situation in which other factors, i.e. other factors than those explicitly figuring in L itself, are held constant).

Recall Earman and Roberts's example of a spurious generalization in which the antecedent is irrelevant for the consequent: "all spherical bodies conduct electricity" (Earman and Roberts 1999, 253). According to Hitchcock and Woodward's definition of minimal invariance, it might as well be that in fact all actual spherical bodies conduct electricity - however the correlation of being spherical and conductivity turns out to be accidental, because one can at least imagine a counterfactual situation in which a the geometrical shape of the body in question is changed from spherical to being cubical by intervention and, yet, the conductivity remains unchanged. Analogously, my causalisolation-version of invariance assumes that the counterfactual "if it were the case that (a) the geometrical shape of the body were cubical (and not spherical, as it actually is) and (b) the body were causally isolated, then the body would conduct electricity differently than it actually does" is false. This evaluation of the counterfactual is, of course, in accord with Earman and Roberts's intuitions. Thus, both versions of invariance theories classify the 
generalization "all spherical bodies conduct electricity" correctly as accidental, because counterfactual changes of the antecedent are irrelevant for a change of the consequent. In other words, being spherical fails to be relevant for conductivity of electricity, because the statement "all spherical bodies conduct electricity" fails to be minimally invariant. ${ }^{22}$

To sum up, quasi-Newtonian laws (i.e. a non-epistemic, objective, ontological version of Pietroski and Rey's ECC) and an account of relevance in terms of invariance are supplements for a theory of lawish statements in the special sciences.

\section{Conclusion: The Explication of Special Science Laws}

I started out by asking how non-universal generalizations in the special science can perform a lawish function. I have distinguished four dimensions of universality. Further, I demanded to develop a theory for each dimension. I argued for preserving the universality in the first and second dimension for laws of the special sciences - taking this into account is still compatible with describing special science laws as system laws (cf. Schurz 2002). The non-universality in the third dimension is taken care of by the Mill-Maudlin-view of quasi-Newtonian laws. We deal with the fourth dimension of non-universality by relying on the notions of minimal invariance. Based on these results, my explication of a special science law is the following:

A statement $\mathrm{L}$ is a special science law iff (1) $\mathrm{L}$ is a system law, (2) $\mathrm{L}$ is quasiNewtonian, and (3) L is minimally invariant.

I argued that this explication has several benefits: (a) it allows to avoid Lange's Dilemma, and, (b) it meets the requirement of relevance in terms of invariance. I dare to conclude

\footnotetext{
22 Cf. Woodward 2003, 2008, Woodward and Hitchcock 2003 for a more detailed account of this invariantist strategy to distinguish lawish statements from accidentally true generalizations. Reutlinger, Hüttemann and Schurz (in print, section 6) provide an easily accessible survey to invariance theories.
} 
that, according to my reconstruction, non-universal special science laws are at least good candidates for true, empirical (i.e. not trivially true) statements playing a lawish role in the special sciences.

\section{Acknowledgements}

I would like to thank Alexander Bird, Luke Glynn, Andreas Hüttemann, Meinard Kuhlmann, James Ladyman, Tim Maudlin, Wolfgang Spohn, Michael Strevens, Emma Tobin, the members of my DFG Research Group "Causation and Explanation", and two anonymous referees of this journal for considerably improving my paper by their stimulating comments.

\section{References}

Armstrong, David. 1983. What Is a Law of Nature?, Cambridge, MA: Cambridge University Press.

Baumgartner, Michael. 2008. "Regularity Theories Reassessed" Philosophia 36, 327-354.

Beatty, John. 1995. “The Evolutionary Contingency Thesis.”, In G. Wolters and G.J.

Lennox (eds.), Concepts, Theories, and Rationality in the Biological Sciences,

Pittsburgh: Pittsburgh University Press, 45-81.

Bechtel, William, and Adele Abrahamsen. 2005. "Explanation: A Mechanist Alternative." Studies in History and Philosophy of Biological and Biomedical Sciences 36: 421441.

Bird, Alexander. 2007. Nature's Metaphysics: Laws and Properties, Oxford: Oxford University Press.

Braithwaite, R.B..1959. Scientific Explanation. Cambridge: Cambridge University Press.

Callender, Craig and Jonathan Cohen. 2010. "Special Sciences, Conspiracy and the Better Best System Account of Lawhood.”, Erkenntnis 73.3: 427-447. 
Cartwright, Nancy. 1983. How the Laws of Physics Lie, Oxford: Oxford University Press.

Cartwright, Nancy. 1989. Nature's Capacities and their Measurement, Oxford: Oxford University Press.

Craver, Carl. 2007. Explaining the Brain. Mechanisms and the Mosaic Unity of

Neuroscience, Oxford: Clarendon Press.

Dowe, Phil. 2000. Physical Causation, Cambridge, MA: Cambridge University Press.

Eagle, Antony. 2010. Philosophy of Probability: Contemporary Readings, London:

Routledge.

Earman, John, and John Roberts. 1999. "Ceteris Paribus, There is no Problem of

Provisos.”, Synthese 118: 439-478.

Earman, J., J. Roberts, and S. Smith. 2002. “Ceteris Paribus Lost”, in Ceteris Paribus laws, J. Earman et al. (eds.), Erkenntnis 52 (Special Issue), pp. 281-301.

Gadenne, Volker. 2004. Philosophie der Psychologie, Bern: Verlag Hans Huber.

Glennan, Stuart. 2002. "Rethinking Mechanistic Explanation." Philosophy of Science 69 (Proceedings): S342-S353.

Hausman, Daniel. 1992. The Separate and Inexact Science of Economics,

Cambridge/MA: Cambridge University Press.

Hitchcock, Christopher. 2001. "The Intransitivity of Causation Revealed in Equations and Graphs.” Journal of Philosophy 98: 273-299.

Hüttemann, Andreas. 2007. "Naturgesetze." In Wissenschaftstheorie, eds. Andreas

Bartels, and Manfred Stöckler, 135-153, Paderborn: mentis.

Hüttemann, Andreas (forthcoming) "Ceteris Paribus Gesetze in der Physik."

Kincaid, Harold. 2004. "Are There Laws in the Social Sciences?: Yes", In Contemporary Debates in the Philosophy of Science, ed. Christopher Hitchcock, 168-187, Oxford: Blackwell.

Kincaid, Harold. 2009. "Explaining Growth.” In Kincaid, H., and D. Ross (eds.), 2009, The Oxford Handbook of Economics, Oxford: Oxford University Press, 455-475. 
Ladyman, James and Don Ross. 2007. Every Thing Must Go. Metaphysics Naturalized, Oxford: Oxford University Press.

Lange, Marc. 1993. "Natural Laws and the Problem of Provisos.” Erkenntnis 38:

233-248.

Lange, Marc. 2000. Natural Laws in Scientific Practice. Oxford: Oxford University Press.

Lange, Marc. 2002. “Who's Afraid of Ceteris Paribus Laws? Or: How I learned to stop worrying and love them.” In Ceteris Paribus laws, J. Earman et al. (eds), Erkenntnis 52 (Special Issue), pp. 407-423.

Lange, Marc. 2009. Laws and Lawmakers. Oxford: Oxford University Press.

Leuridan, Bert. 2010. “Can Mechanisms Really Replace Laws of Nature?.” Philosophy of Science 77, 317-340.

Lewis, David 1973a. Counterfactuals. Oxford: Blackwell.

Lewis, David. 1973b. “Causation.” In Philosophical Papers, by David Lewis, 159-171,

Oxford: Oxford University Press.

Lewis, David. 1986. “Causal explanation.” In Philosophical Papers, volume 2, Oxford:

Oxford University Press, pp. 214-240.

Loewer, Barry. 2008. "Why There Is Anything Except Physics." In Being Reduced. New Essays on Reduction, Explanation, and Causation, eds. Jakob Hohwy and Jesper Kallestrup, pp. 149-163, Oxford: Oxford University Press.

Machamer, Peter, Lindley Darden, and Carl F. Craver. 2000. "Thinking about

Mechanisms." Philosophy of Science 67: 1-25.

Mackie, John L.. 1974. The Cement of the Universe. Oxford: Oxford University Press.

Mas-Colell, A., M. Whinston, and J. Green, 1995, Microeconomic Theory, Oxford: Oxford University Press.

Maudlin, Tim. 2004. "Causation, Counteractuals, and the Third Factor", In Causation and Counterfactuals, eds. Ned Hall et al., 419-444, Cambrigde, Ma: MIT Press.

Maudlin, Tim. 2007. The Metaphysics Within Physics, Oxford: Oxford University Press. 
Mill, J. S.. 1836/2008. “On the Definition and Method of Political Economy.” In The

Philosophy of Economics. An Anthology, ed. Daniel Hausman, 41-58, New York:

Cambridge University Press, Third Edition.

Mitchell, Sandra. 1997. "Pragmatic Laws.” Philosophy of Science, 64: 242-265.

Mitchell, Sandra. 2000. "Dimensions of Scientific Law.” Philosophy of Science 67: S468S479.

Mitchell, Sandra. 2002. “Ceteris Paribus - An Inadequate representation of biological

Contingency", in Ceteris Paribus laws, J. Earman et al. (eds), Erkenntnis 52 (Special Issue), pp. 329-350.

Mumford, Stephen. Laws in Nature, London: Routledge.

Pearl, Judea. 2000. Causality: Models, Reasoning and Inference, Cambridge: Cambridge University Press.

Pietroski, Paul, and Georges Rey. 1995. "When other things aren’t equal: Saving Ceteris Paribus Laws from Vacuity.” British Journal for the Philosophy of Science 46: 81110.

Reutlinger. Alexander. in print. "What's Wrong with the Pragmatic-Ontic Account of Mechanistic Explanation?", In Explanation, Prediction, and Confirmation, edited by Dennis Dieks, Wenceslao J. Gonzalez, Stephan Hartmann, Thomas Uebel, Marcel Weber, Dordrecht: Springer.

Reutlinger, Alexander. Forthcoming. "Is Non-Reductive Conceptual Analysis A MetaPhilosophical Problem for Theories of Causation?", Logique et Analyse.

Reutlinger, Alexander, Andreas Hüttemann and Gerhard Schurz. in print. "Ceteris paribus Laws, The Stanford Encyclopedia of Philosophy.

Roberts, John. 2004. "There are No Laws in the Social Sciences.” In Contemporary

Debates in the Philosophy of Science, ed. Christopher Hitchcock, 168-185, Oxford:

Blackwell. 
Rosenberg, Alexander. 2001. "How is biological explanation possible?.", British Journal for Philosophy of Science 52, 735-760.

Rosenberg, Alexander and Daniel W. McShea. 2008. Philosophy of Biology. A

Contemporary Introduction, Routledge: London.

Schurz, Gerhard. 2001. "Pietroski and Rey on Ceteris Paribus Laws." British Journal for Philosophy of Science 52: 359-370.

Schurz, Gerhard. 2002. "Ceteris Paribus Laws: Classification and Deconstruction." In

Ceteris Paribus Laws, eds. John Earman et al., 351-372, Dordrecht: Kluwer.

Schurz, Gerhard. 2005. “Laws of Nature versus System Laws.” In Nature’s Principles, eds. J. Faye et al., 255-268, Dordrecht: Kluwer.

Sober, Elliott. 1997. "Two Outbreaks of Lawlessness in Recent Philosophy of Biology." Philosophy of Science 64: 432-444.

Strevens. Michael. 2007. "Review of Woodward, Making Things Happen." Philosophy and Phenomenological Research LXXIV, No. 1, 233-249.

Strevens. Michael. 2008. "Comments on Woodward, Making Things Happen.” Philosophy and Phenomenological Research, Vol. LXXVII No. 1, 171-192.

Strevens, Michael. 2009. Depth. An Account of Scientific Explanation, Cambridge, MA: Harvard University Press.

Strevens, Michael. 2010. “Ceteris Paribus Hedges: Causal Voodoo That Works”, U R L = $<$ http://www.strevens.org/research/lawmech/CPMechBrev.pdf $>$.

Tooley, Michael. 1977. “The Nature of Laws.” Canadian Journal of Philosophy 7: 667-98.

Weber, Marcel. 2008. "Causes Without Mechanisms. Experimental Regularities, Physical Laws, and Neuroscientific Explanation.” Philosophy of Science 75, 995-1007.

Woodward, James. 2002. "There is no such thing as a ceteris paribus law." In Ceteris Paribus laws, John Earman et al. (eds), 303-328, Erkenntnis 52 (Special Issue).

Woodward, James. 2003. Making Things Happen. Oxford: Oxford University Press.

Woodward, James. 2007. "Causation with a Human Face.”, In Causation, Physics, and the 
Constitution of Reality. Russell's Republic Revisited, H. Price and R. Corry (eds.), Oxford: Clarendon Press, pp. 66-105.

Woodward, James. 2008. "Response to Strevens." Philosophy and Phenomenological Research, Vol. LXXVII No. 1, 193-212.

Woodward, James, and Christopher Hitchcock. 2003. "Explanatory Generalizations, Part I: A Counterfactual Account.” Nous 37.1: 1-24. 